RESEARCH ARTICLE

\title{
Time-of-flight methodologies with large-area diamond detectors for ion characterization in laser-driven experiments
}

\author{
M. Salvadori@ ${ }^{1,2}$, G. Di Giorgio ${ }^{1}$, M. Cipriani ${ }^{1}$, M. Scisciò ${ }^{1}$, C. Verona ${ }^{3}$, P. L. Andreoli ${ }^{1}$, G. Cristofari ${ }^{1}$, \\ R. De Angelis ${ }^{1}$, M. Pillon ${ }^{1}$, N. E. Andreev ${ }^{4}$, P. Antici ${ }^{2}$, N. G. Borisenko ${ }^{5}$, D. Giulietti ${ }^{6,7}$, M. Migliorati ${ }^{8,9}$, \\ O. Rosmej ${ }^{10,11}$, S. Zähter ${ }^{10,11}$, and F. Consoli ${ }^{1}$ \\ ${ }^{1}$ Fusion and Nuclear Safety Department, ENEA, Frascati, Italy \\ ${ }^{2}$ INRS-EMT, Varennes, QC, Canada \\ ${ }^{3}$ Industrial Engineering Department, University of Rome "Tor Vergata", Rome, Italy \\ ${ }^{4}$ Joint Institute for High Temperatures, RAS, Moscow, Russia \\ ${ }^{5}$ P. N. Lebedev Physical Institute, RAS, Moscow, Russia \\ ${ }^{6}$ Department of Physics, University of Pisa, Pisa, Italy \\ ${ }^{7}$ INFN of Pisa, Pisa, Italy \\ ${ }^{8}$ University of Rome "La Sapienza”, Rome, Italy \\ ${ }^{9}$ INFN of Rome, Rome, Italy \\ ${ }^{10}$ GSI Helmotzzentrum für Schwerionenforschung GmbH, Darmstadt, Germany \\ ${ }^{11}$ Goethe University Frankfurt, Frankfurt, Germany \\ (Received 1 August 2021; revised 21 December 2021; accepted 27 December 2021)
}

\begin{abstract}
The time-of-flight technique coupled with semiconductor detectors is a powerful instrument to provide real-time characterization of ions accelerated because of laser-matter interactions. Nevertheless, the presence of strong electromagnetic pulses (EMPs) generated during the interactions can severely hinder its employment. For this reason, the diagnostic system must be designed to have high EMP shielding. Here we present a new advanced prototype of detector, developed at ENEA-Centro Ricerche Frascati (Italy), with a large-area $(15 \mathrm{~mm} \times 15 \mathrm{~mm})$ polycrystalline diamond sensor having $150 \mu \mathrm{m}$ thickness. The tailored detector design and testing ensure high sensitivity and, thanks to the fast temporal response, high-energy resolution of the reconstructed ion spectrum. The detector was offline calibrated and then successfully tested during an experimental campaign carried out at the PHELIX laser facility $\left(E_{L} \sim 100 \mathrm{~J}\right.$, $\left.\tau_{L}=750 \mathrm{fs}, I_{L} \sim(1-2.5) \times 10^{19} \mathrm{~W} / \mathrm{cm}^{2}\right)$ at GSI (Germany). The high rejection to EMP fields was demonstrated and suitable calibrated spectra of the accelerated protons were obtained.
\end{abstract}

Keywords: diamond detector; ion diagnostics; laser-matter interaction; time of flight

\section{Introduction}

Semiconductor detectors characterized by a wide bandgap, that is, silicon carbide and diamonds, are commonly used as time-resolved sensors for time-of-flight (TOF)

Correspondence to: M. Salvadori and F. Consoli, Fusion and Nuclear Safety Department, ENEA, Frascati, Italy. Email: Martina.salvadori@uniroma1.it (M. Salvadori),fabrizio.consoli@enea.it (F. Consoli) measurements ${ }^{[1-8]}$. This technique is a valuable instrument for the real-time characterization of charged particles accelerated during laser-plasma interaction ${ }^{[9]}$. In particular, when coupled to semiconductor detectors, it allows one to retrieve the particle energy distribution with a good resolution (according to the temporal response of the detector, tolerances of the order of about $2 \%$ are achieved), and to reconstruct the associated spectra with high accuracy ${ }^{[8,10,11]}$. The latter can be retrieved by exploiting 


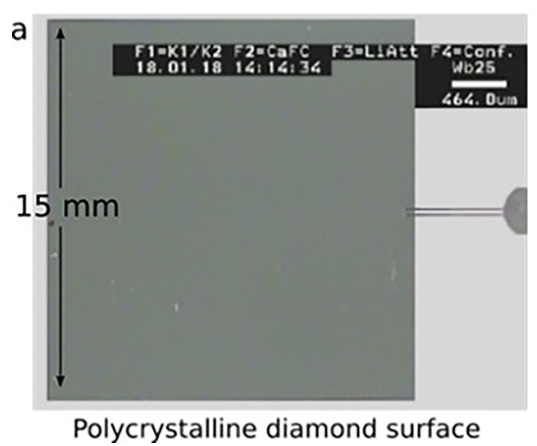

b

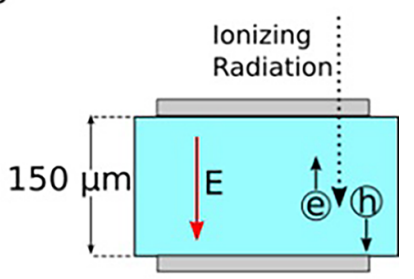

Polycrystalline diamond surface

Figure 1. (a) A picture of the diamond surface taken with a Leica Wild M8 microscope equipped with the charge-coupled device camera JVC TK-C1480B. (b) A scheme of the diamond detector structure. The $150 \mu \mathrm{m}$ polycrystalline diamond wafer is enclosed between two electrodes (made of $4 \mathrm{~nm}$ DLC, $4 \mathrm{~nm}$ $\mathrm{Pt}$ and $200 \mathrm{~nm} \mathrm{Au}$ ) providing a constant electric field allowing for charge collection.

the working principle of semiconductor detectors. When ionizing radiation interacts with the bulk of the material, it releases its energy and produces a certain number of free electron-hole pairs, accordingly ${ }^{[12]}$. These charges are then collected at the electrodes generating the signal. Knowing the detector response and the ion type, it is possible to retrieve the number of particles impinging onto the detector for each energy range ${ }^{[8,13]}$.

The very high purity and crystalline quality of single crystal diamonds guarantee a considerably high value of charge carrier mobility and charge collection efficiency (CCE; up to 98\%), resulting in TOF measurements characterized by a high temporal resolution (down to $\sim 0.5 \mathrm{~ns}$ ), thus allowing one to retrieve spectra with remarkable energy resolution ${ }^{[8,12,13]}$. Nevertheless, single crystal diamonds can be commonly produced only with small surfaces (up to $5 \mathrm{~mm} \times 5 \mathrm{~mm}$ ) ${ }^{[12]}$. In experiments of laser-matter interaction, this limits the solid angle of detection coverage, and thus the overall sensitivity of the detection system. Indeed, there are several applications, for instance those characterized by low fluxes of emitted particles, where instead high sensitivities are required ${ }^{[14]}$. For other cases, because the ion fluxes decrease with respect to energy, information about the maximum energy of the emitted particles can be retrieved accurately only by detectors with high sensitivity. For this purpose, a polycrystalline diamond structure of high quality offers the possibility to produce sensors characterized by a large area, achieving higher sensitivity while maintaining a good temporal resolution. In this work a prototype of a new detector developed at ENEA-Centro Ricerche Frascati (Italy), based on a commercial $150 \mu \mathrm{m}$ thick high-quality (II-a electronic grade) polycrystalline diamond structure, provided by Diamond Detectors Ltd. (UK), is presented. The sensor, shown in Figure 1(a), has a wide surface $(15 \mathrm{~mm} \times 15 \mathrm{~mm})$. This allows one to increase the overall detection area by about one order of magnitude with respect to classical $5 \mathrm{~mm} \times 5 \mathrm{~mm}$ monocrystalline detectors $^{[5,7,8,13,15]}$, covering a larger solid angle and enhancing the overall sensitivity of the diagnostic system. As shown in Figure 1(b), the diamond wafer is enclosed between two metallic electrodes (consisting of $4 \mathrm{~nm}$ diamond-like carbon (DLC), $4 \mathrm{~nm}$ of platinum and $200 \mathrm{~nm}$ of gold) placed on its large surface that, when biased, provide a constant electric field throughout the diamond thickness, allowing for efficient charge collection and eventually ion beam characterization.

When a high-intensity laser $\left(>10^{16} \mathrm{~W} / \mathrm{cm}^{2}\right)$ interacts with matter, transient electromagnetic waves (spanning over hundreds of nanoseconds after the interaction) of remarkable intensity (up to the $\mathrm{MV} / \mathrm{m}$ order) in the radiofrequencymicrowave range are produced ${ }^{[16,17]}$. The presence of these strong electromagnetic pulses (EMPs) is a serious threat for electronic devices placed near the interaction point. Indeed, for classical TOF ion diagnostics, EMPs can couple both with the detection system and the acquisition system, often heavily hindering the effective measurement of accelerated ions ${ }^{[15]}$. To avoid this effect, an advanced TOF procedure has already been developed ${ }^{[8,15]}$. This offers a good rejection to the EMPs and allows for the suitable spectral representation of the detected protons. The employment of a large-area sensor significantly improves the detector sensitivity but, on the other hand, makes the detection system more prone to coupling with EMPs. Particular attention has been thus dedicated to the design of an advanced housing of the sensor, with the purpose of achieving high rejection to EMPs. This will allow it to be fruitfully employed even in the typical harsh environments of experiments that involve high-energy lasers interacting with matter, with the future purpose to successfully employ this type of detector in the new facilities under development around the world, such as Apollon in France $\left(\tau_{L}=15 \mathrm{fs}, E_{L}=150 \mathrm{~J}, 10 \mathrm{PW}\right)$ and L4 ATON in Czech Republic ( $\left.\tau_{L}=150 \mathrm{fs}, E_{L}=1.5 \mathrm{~kJ}, 10 \mathrm{PW}\right)$.

In the following sections we will first introduce the basic concepts of the TOF technique coupled to semiconductor detectors. The novel detector design is then accurately described together with the performed characterization procedure. Finally, the results obtained during an experimental 
campaign, where remarkable levels of EMPs were produced, are presented and discussed.

\section{Working principle}

\subsection{Time-of-flight technique and spectrum reconstruction}

The TOF technique relies on the measurement of the time required by a particle for traveling over a known distance $d_{\text {TOF. }}$ Particles emitted from the source are collected by means of a time-resolved detector. The velocity distribution of the particles is thus transformed to a temporal distribution $^{[18]}$. Since ionizing photons (UV-X) are also emitted during the interaction, their detection instant, $t_{\mathrm{ph}}$, is used as an absolute reference, on the same oscilloscope trace, to retrieve the actual interaction instant $t_{\text {bang }}{ }^{[8]}$, namely:

$$
t_{\text {bang }}=t_{\mathrm{ph}}-\frac{d_{\mathrm{TOF}}}{c},
$$

where $c$ is the speed of light in vacuum. The energy of the particles generating the signal can be then retrieved by using the classical relativistic relation:

$$
E_{i}=m_{i}\left(\gamma_{i}-1\right) c^{2}
$$

where $m_{i}$ is the ion mass and $\gamma_{i}=\left(1-\frac{v_{i}^{2}}{c^{2}}\right)^{-\frac{1}{2}}=$ $\left(1-\frac{d_{\mathrm{TOF}}^{2}}{c^{2}\left(t_{i}-t_{\mathrm{bang}}\right)^{2}}\right)^{-1 / 2}$ is the relativistic parameter.

Actually, ion energies typically achieved in laser-plasma experiments are well below the relativistic limit; proton energies up to $10 \mathrm{MeV}$ are routinely achievable with most laser systems, whereas only in the last years few tens of $\mathrm{MeV}$ have become more and more easily accessible, and the current record in terms of maximum proton energy is $94 \mathrm{MeV}^{[19]}$. Thus, the previous relation simplifies to $E_{i} \simeq$ $\frac{1}{2} m_{i} v_{i}^{2}$. Indeed, for protons up to approximately $10 \mathrm{MeV}$, $\beta \leq 0.15$ and the error in considering the classical expression instead of the relativistic one is lower than $2 \%$.

Once the energy is known, from the amplitude of the signal, it is possible to obtain the number of particles impinging onto the detector by using the general relation ${ }^{[8,15]}$ :

$$
N_{i}=\frac{Q_{c} \epsilon_{g}}{q_{e}} \frac{1}{E_{i} \mathrm{CCE}},
$$

where $q_{e}$ is the electronic charge, $\epsilon_{g}$ is the radiationionization energy, that is, the average energy needed to create a free electron-hole pair inside the detector in use; for diamond detectors it is $13.1 \mathrm{eV}^{[12]}$. CCE is the charge collection efficiency of the detector ${ }^{[12]}$ and $Q_{c}$ is the amount of collected charge that can be estimated by performing a numerical integration of the detected signal $V(t)^{[7,8]}$ :

$$
Q_{c}=k_{A} \int_{t_{i}}^{t_{f}} V(t) \mathrm{d} t .
$$

Here, $k_{A}=\frac{A}{R}$, where $R$ is the load impedance of the acquiring system and $A$ is the link attenuation. The time step to perform the integration is determined by the temporal resolution of the detection system, which is strongly dependent on the time response of the detector in use.

From the previous considerations it is therefore clear that for the spectrum estimation it is necessary to know the actual detector characteristics in terms of temporal response and CCE. For this purpose, the polycrystalline diamond was characterized by exposure to monochromatic $\alpha$ particles having $E_{\alpha}=5.486 \mathrm{MeV}$, emitted by the ${ }^{241} \mathrm{Am}$ radioactive source.

\subsection{Time response and charge collection efficiency measurements}

To appreciate the temporal response by single particle detection, the output of the diamond detector was connected to the biasing fast amplifier model Cividec (nominal gain $G=40 \mathrm{~dB}$, bandwidth $=2 \mathrm{GHz}$ ) and to the LeCroy 620 $\mathrm{Zi}$ scope (bandwidth $=2 \mathrm{GHz}$, sample rate $=20 \mathrm{GS} / \mathrm{s}$ ). The fast amplifier was necessary to both bias the detector and to increase the amplitude of the fast-varying signals to levels detectable by the scope.

The signal shown in Figure 2 has been obtained by averaging over several acquisitions performed with the detector biased at $+250 \mathrm{~V}$, typical bias used to feed the detector. The value of the applied voltage was chosen in order to use the sensor in the velocity saturation regime, that is, when the carrier velocity does not increase for a further increment of the suffered electric field ${ }^{[12]}$.

From this kind of signal it is possible to retrieve the detector temporal response by estimating its full width at half maximum (FWHM), which resulted to be $4.1 \mathrm{~ns}$.

The CCE was estimated by exposing the detector to the ${ }^{241} \mathrm{Am}$ sample for $300 \mathrm{~s}$. Thus, several events were detected by the diamond detector during the acquisition time. The output signals were processed by a conventional chargesensitive electronic chain ${ }^{[12,13,20]}$. They were first integrated by a charge-sensitive preamplifier (ORTEC 142A). Then, the resulting voltage pulse was sent to a shaping amplifier (ORTEC 671) with gain and shaping time fixed to 200 and $6 \mu \mathrm{s}$, respectively. Finally, a multichannel analyzer connected to a computer sorted the input pulses in different bins according to their amplitude, given by the amount of collected charges, allowing one to obtain a pulse height spectrum (PHS). The spectrum acquired by the diamond detector is reported in Figure 3.

The 100\% CCE bin corresponds to a collected charge equal to the number of charges ideally expected in the 


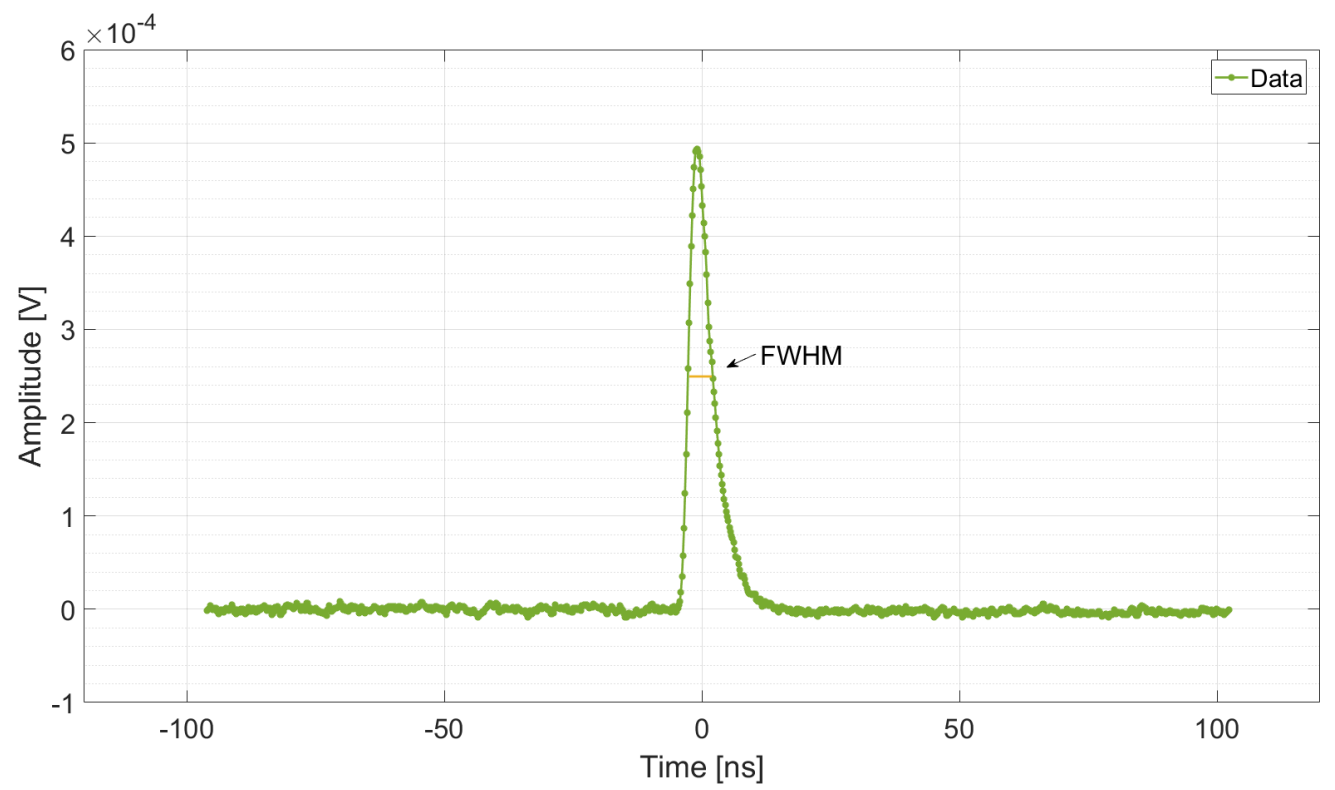

Figure 2. The typical signal provided by the polycrystalline diamond when exposed to single $\alpha$ particles of $5.486 \mathrm{MeV}$. This was obtained by averaging over approximately 100 acquisitions and was used to retrieve the temporal response of the detector $(4.1 \mathrm{~ns})$.

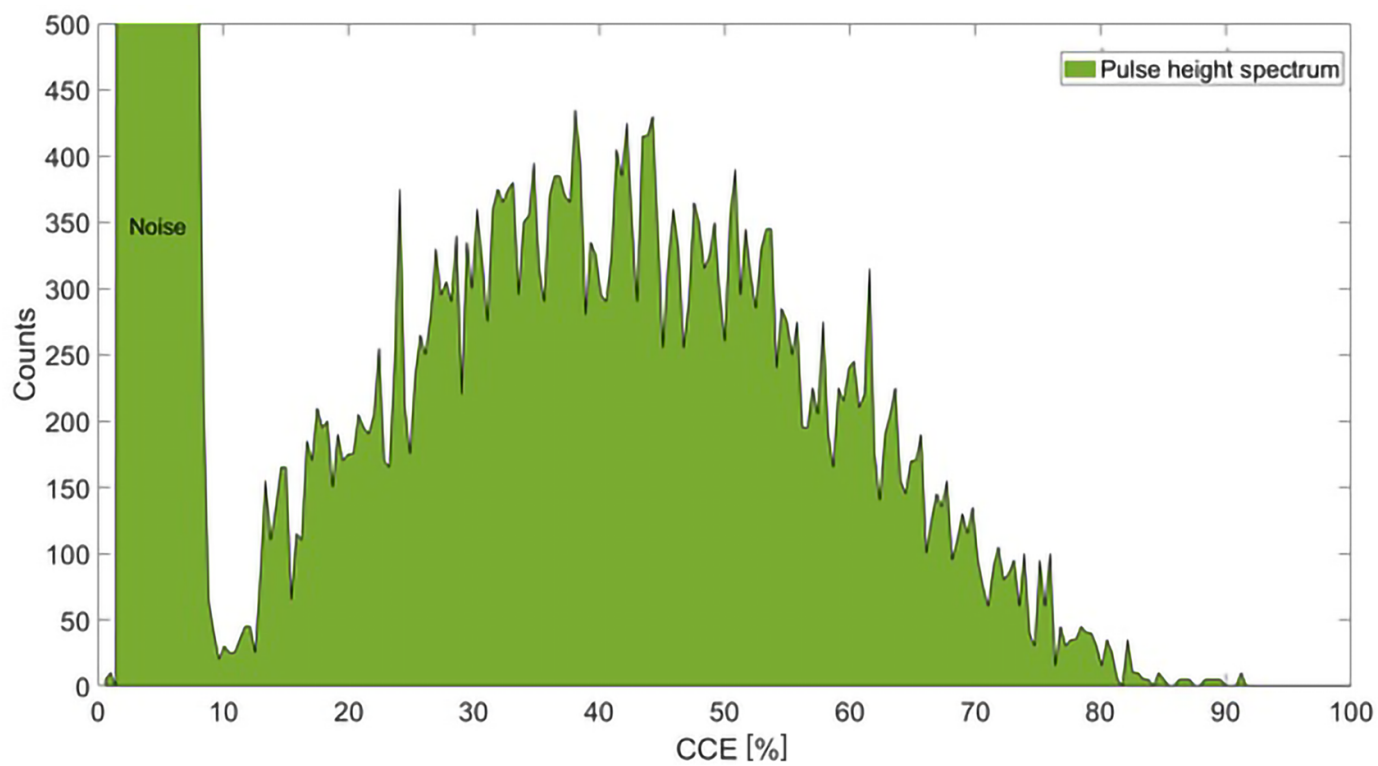

Figure 3. The pulse height spectrum obtained after $300 \mathrm{~s}$ of exposure to the ${ }^{241}$ Am radioactive sample. The estimated charge collection efficiency is approximately $42 \% \pm 21 \%$.

detector for a single incoming $\alpha$ particle, which can be computed by exploiting the following relation ${ }^{[12]}$ :

$$
Q_{g}=\frac{q_{e} E_{\alpha}}{\epsilon_{g}}=6.7 \times 10^{-14} \mathrm{C}
$$

Therefore, an ideal detector would produce a delta-like signal, peaked on the $100 \%$ bin, meaning that all the events produce the same amount of collected charge. Nevertheless, in the case of the polycrystalline diamond detector a broader spectrum is obtained with the peak shifted to lower values of CCE. This effect is due to several factors: trapping recombination centers, dislocations and grain boundary defects of polycrystalline diamond as well as fluctuations of the charge generation process, as reported in the literature ${ }^{[21-23]}$.

The broadening of the spectrum means that even when detecting particles having the same energy that should generate the same number of charges inside the detector, the value of the collected charges varies. From the FWHM of the PHS it is therefore possible to infer the variability of the measured CCE. In this case a CCE value of approximately $42 \%$ with a tolerance of $21 \%$ was estimated. 
a

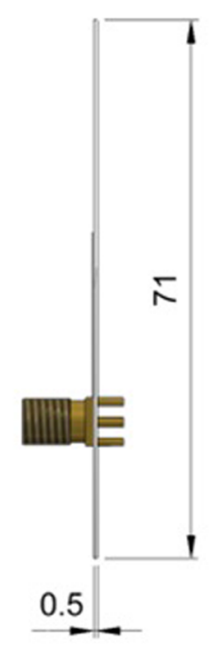

15

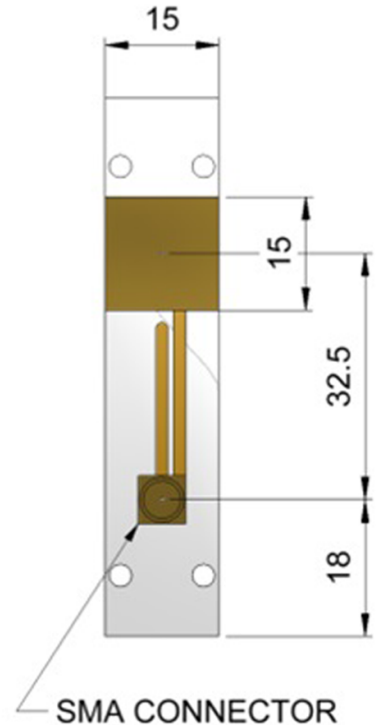

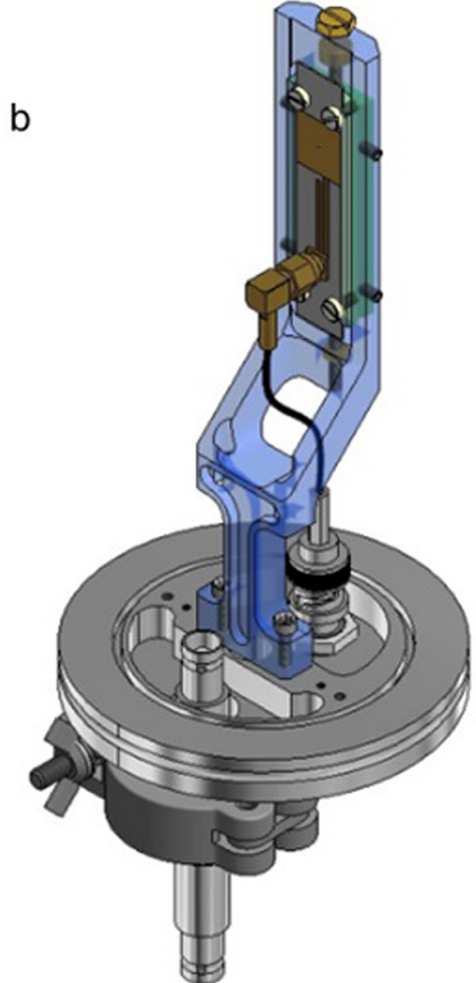

Figure 4. The inner detector layout: (a) the diamond wafer mounted on a thin strip together with the electrical connection, where the bias to the electrodes is sent through an SMA connector; (b) the strip with the polycrystalline diamond detector is mounted on a support that allows its vertical alignment and is then fixed onto a stainless steel cap provided with SMA to BNC feedthroughs for vacuum.

In a previous work ${ }^{[13]}$ the response of two typical single crystal diamond detectors presenting different electrodes layouts was characterized by using this methodology. The diamond with sandwich electrode configuration gave an almost ideal response with CCE $(98 \pm 2) \%$, whereas the interdigital electrodes layout showed a lower and broader CCE $(68 \pm 18) \%$.

\subsection{Sensitivity enhancement estimation}

The improved sensitivity of this detector with respect to the mentioned typical single crystal structures is due to the wider surface of the detector itself. This allows one to cover a larger solid angle, resulting in an overall greater sensitivity of the detection system.

In a TOF scheme, the sensitivity is given by the capability of the detector to supply a signal with intensity high enough to be effectively detected by the scope used for the measurement, according also to its noise floor.

A quality factor to express the actual sensitivity of the detector in schemes of laser-matter interaction could be defined as the product of CCE per detector area.

This relation allows for inferring the actual improvement in sensitivity of the polycrystalline by computing the ratio $\frac{\mathrm{CCE}_{\text {polycrystalline }} \text { Area }_{\text {polycrystalline }}}{\mathrm{CCE}_{\text {singlecrystal }}{ }^{\text {Area }} \text { }_{\text {singlecrystal }}}$.
As already mentioned, single crystal diamond detectors have higher CCE $(68 \%-98 \%)$ but lower area $(5 \mathrm{~mm} \times$ $5 \mathrm{~mm}$ ). According to data supplied in the previous section, the achieved sensitivity improvement by using the wide area polycrystalline diamond detector is in the range of 3.9-5.7, depending on the specific single crystal detector considered for the comparison.

\subsection{EMP shielding}

To be effectively employed in environments characterized by high levels of EMPs, the diamond sensor was mounted in a case optimized for optimal EMP rejection.

As shown in Figure 4(a), the diamond detector is located on a thin strip where all the electrical connections are built-in. In the developed detector design, these electrical connections are kept at a minimal length and are provided with an optimized grounding system and double-shielded cabling. The strip is then fixed on an ad hoc support that allows for its vertical alignment and mounting it onto a stainless steel cap (Figure 4(b)).

The whole assembly is then inserted into the final holder. This consists of two cylindrical structures, one containing the other. In Figures 5(a) and 5(b), it is possible to see that the assembly hosting the diamond detector fits into the inner cylinder that, when properly closed, works as a Faraday 

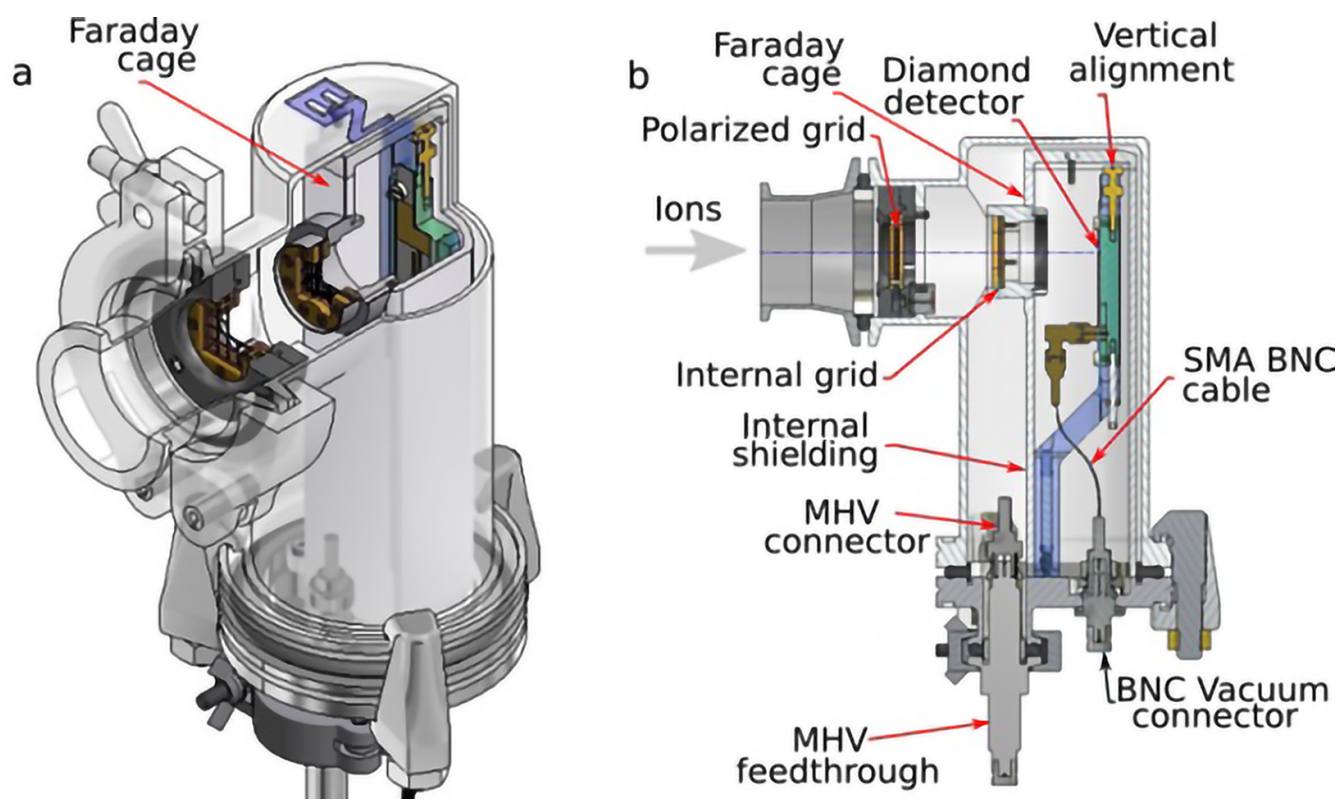

Figure 5. (a) The external case in which the support shown in Figure 4(b) is mounted. It is possible to see the inner Faraday cage and the two grids. (b) The scheme of the whole detector layout.

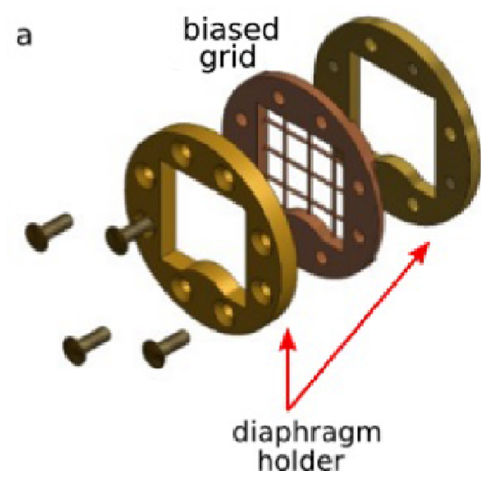

b

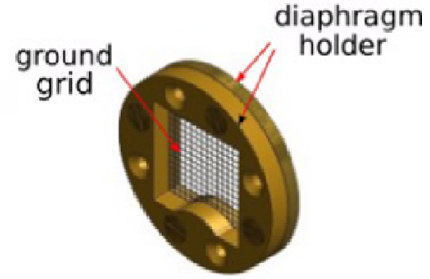

Figure 6. The two copper grids placed in front of the diamond detector: (a) the polarized grid can be biased up to $5 \mathrm{kV}$ and has a mesh with a $4.5 \mathrm{~mm}$ step; (b) the grounded grid is characterized by a denser mesh ( $2 \mathrm{~mm}$ step), which is suitable for effective closing of the inner Faraday cage.

cage, providing an effective shielding to the detector from the external environment. The closure of the cage can be provided either with a thin metallic sheet or with a metallic grid designed with appropriate geometrical characteristics suitable for the shielding from the external electromagnetic waves.

Indeed, the detector holder is designed to allow the positioning of two grids mounted parallel to each other and to the diamond, as it is possible to see in Figures 5(a) and 5 (b). Both the grids are made of copper and the diameter of the wires used for the mesh is $0.5 \mathrm{~mm}$. The distance between the two grids and the distance between internal grid and diamond sensor are both of $40 \mathrm{~mm}$. These values were due to mechanical and electrical reasons in the device development, to get reliable working operations. The first grid, shown in Figure 6(a), can be biased with voltages up to $5 \mathrm{kV}$ through an internal cable with an MHV connector.
The step of the mesh is $4.5 \mathrm{~mm}$. This grid is isolated from the rest of the structure through a thermoplastic support made of white Delrin. The second internal grid, shown in Figure 6(b), is characterized by a denser wire mesh having a step of $2 \mathrm{~mm}$. The grid material and geometrical parameters are chosen to be effectively used as closing components of the internal Faraday cage. Indeed, this grid is directly connected to the ground of the structure, and it is an integral part of the internal metallic shield, as can be seen in Figures 5(a) and $5(\mathrm{~b})$.

We designed the grids by taking into account Ref. [24]. The internal grid (with $2 \mathrm{~mm}$ mesh step), meant for the actual electromagnetic shielding of the diamond, was designed to have transmission lower than $100 \mathrm{~dB}$ up to $10 \mathrm{GHz}$. This is the frequency range where EMP fields are known to be more intense ${ }^{[16]}$. The external grid, with a $4.5 \mathrm{~mm}$ mesh step, is also capable of giving good shielding, but it has been rather 
designed to have high particle transparency and yet to be robust for maintaining suitable biasing when required.

When the external grid is biased, the two-grid assembly provides a static electric field, which is useful to separate the electron contribution from the ionic component. As mentioned, the maximum biasing voltage is $5 \mathrm{kV}$, allowing electrons up to about $5 \mathrm{keV}$ to be inhibited to reach the diamond. In this way ions with mass $m_{i}$ and energies up to $E_{\max }=5 \mathrm{keV} \times\left(m_{i} / m_{e}\right)$, where $m_{e}$ is the electron mass, will be separated from the co-moving electrons.

The dimensions of the two squared grids are $18 \mathrm{~mm}$ $\times 18 \mathrm{~mm}$ (the external one) and $16 \mathrm{~mm} \times 16 \mathrm{~mm}$ (the internal one). So, both are larger than the actual diamond. For particles having penetration ranges in $\mathrm{Cu}$ lower than the wire diameter, the employment of both of these grids would reduce the actual active surface of the diamond by a factor of approximately $25 \%$, due to the area covered by the projection of the wires of the two grids on the diamond plane. Nevertheless, thanks to the large area of the polycrystalline diamond, this reduction does not remarkably affect the overall detector sensitivity and allows one to use the detector also without the necessity to use any further conductive shielding foil. However, the holder foresees the possibility to replace the inner grid with a thin foil filter right in front of the diamond detector, in order to cut the TOF contribution given by heavy ion detection, when necessary ${ }^{[8]}$. The combined action of the biased grid and the filter allows only protons to reach the detector, leading to an easier spectrum reconstruction.

All of these components are located in the external cylindrical case, made of stainless steel with walls having $2 \mathrm{~mm}$ thickness. These walls provide an additional shielding for electromagnetic waves with frequency down to approximately $500 \mathrm{~Hz}$, exploiting the electromagnetic skin effect ${ }^{[25]}$.

\section{Experimental results}

The performances of the described detector were successfully tested during an experimental campaign carried out at the PHELIX laser facility (GSI, Germany) ${ }^{[26,27]}$. During the experiment two laser pulses were combined to study the effect of controlled pre-plasma on laser-driven acceleration. A hydrodynamically stable, long scale-length near-critical density plasma was generated by irradiating low-density polymer foams with a nanosecond pulse kept at an intensity of approximately $5 \times 10^{13} \mathrm{~W} / \mathrm{cm}^{2}$. Then, with a delay of $2-$ $3 \mathrm{~ns}$, a short pulse of $750 \mathrm{fs}$ having $100 \mathrm{~J}$ energy before compression, was used to irradiate this plasma, delivering up to approximately $20 \mathrm{~J}$ on target in an elliptical focal spot with FWHM diameters of $18 \pm 2 \mu \mathrm{m}$ and $12 \pm 2 \mu \mathrm{m}$ reaching an intensity of approximately $(1-2.5) \times 10^{19} \mathrm{~W} / \mathrm{cm}^{2[28]}$. The nanosecond pulse was used to trigger a super-sonic ionization wave and to prepare the near-critical density plasma for the subsequent main pulse. This was achieved with a longfocusing parabola having a focal length of $150 \mathrm{~cm}$, which provided a long Rayleigh length keeping the intensity of the ns-pulse constant inside the entire thickness of the foam target ${ }^{[28]}$. The same long-focusing parabola was used also for the main pulse, resulting in the mentioned laser intensity of the $10^{19} \mathrm{~W} / \mathrm{cm}^{2}$ order.

This configuration, including the short and energetic laser pulse, is recognized to be one of the main scenarios where the produced EMP fields are most intense ${ }^{[16]}$. Thus, it represented a very good test to prove the EMP rejection of the developed detector assembly.

The EMP level was monitored by means of a custom D-Dot differential electric-field probe ${ }^{[16]}$. In Figure 7 the EMP spectrum for typical shot conditions is reported. It was acquired by a D-Dot probe placed behind a $10 \mathrm{~cm}$ thick Teflon shield at about $120 \mathrm{~cm}$ from the interaction point, where a cellulose triacetate (TAC, $\mathrm{C}_{12} \mathrm{H}_{16} \mathrm{O}_{8}$ ) cylindrical foam target (thickness $=425 \mu \mathrm{m}$, density $2 \mathrm{mg} / \mathrm{cm}^{3}$ ) fixed inside a Cu-washer of $2.5 \mathrm{~mm}$ in diameter ${ }^{[29]}$ was irradiated with a first ns laser pulse followed, after $3 \mathrm{~ns}$, by the $750 \mathrm{fs}$ laser pulse having energy of $82.4 \mathrm{~J}$ (delivering on target 14.6 J). During this shot, the associated electric field estimated by the procedure reported in Ref. [30] exceeded the value of $100 \mathrm{kV} / \mathrm{m}$. The signal covered the whole bandwidth of the scope used for the measurement (Lecroy $735 \mathrm{Zi}$, $3.5 \mathrm{GHz}$ bandwidth $)^{[31]}$.

During a shot performed in similar conditions (for this shot the energy of the $750 \mathrm{fs}$ laser pulse before compression was $86.1 \mathrm{~J}$ and the TAC foam thickness was $425 \mu \mathrm{m}$ ), the polycrystalline diamond detector provided the signal shown in Figure 8. The detector was biased at $+300 \mathrm{~V}$ and placed on the horizontal plane at $90 \mathrm{~cm}$ from the interaction point at 37 degrees from the target normal axis. The signal provided by the diamond was collected by means of the Lecroy HDO 8108 scope (bandwidth $=1 \mathrm{GHz}$, effective number of bits $(\mathrm{ENOB})=8.6$, sampling rate $=10 \mathrm{GS} / \mathrm{s})$.

During this shot the diamond detector was covered with a $20 \mu \mathrm{m}$ thick aluminum filter. This allowed to cut the heavier ion contribution at the expense of the loss of information in the lower energy region of the proton spectrum (below $\sim 1.2 \mathrm{MeV}$ ).

The collected signal clearly presents a high signal-tonoise ratio, estimated to be $17.6 \mathrm{~dB}$. The latter was inferred by comparing this signal with one acquired on a different channel of the same scope closed on a $50 \Omega$ termination that allowed us to estimate the noise level due to the EMP coupling with the scope. The signal shown in Figure 8 was acquired with the channel scale set on $1 \mathrm{~V} / \mathrm{div}$, whereas the channel monitoring the noise level was set on $500 \mathrm{mV} / \mathrm{div}$. In the signal, the temporal position of the photopeak is clearly recognizable as well as the instant when the protons with maximum energy are detected.

If necessary, to enhance the EMP shielding effectiveness while increasing the temporal resolution of the system, the TOF line can be extended by means of an aluminum 

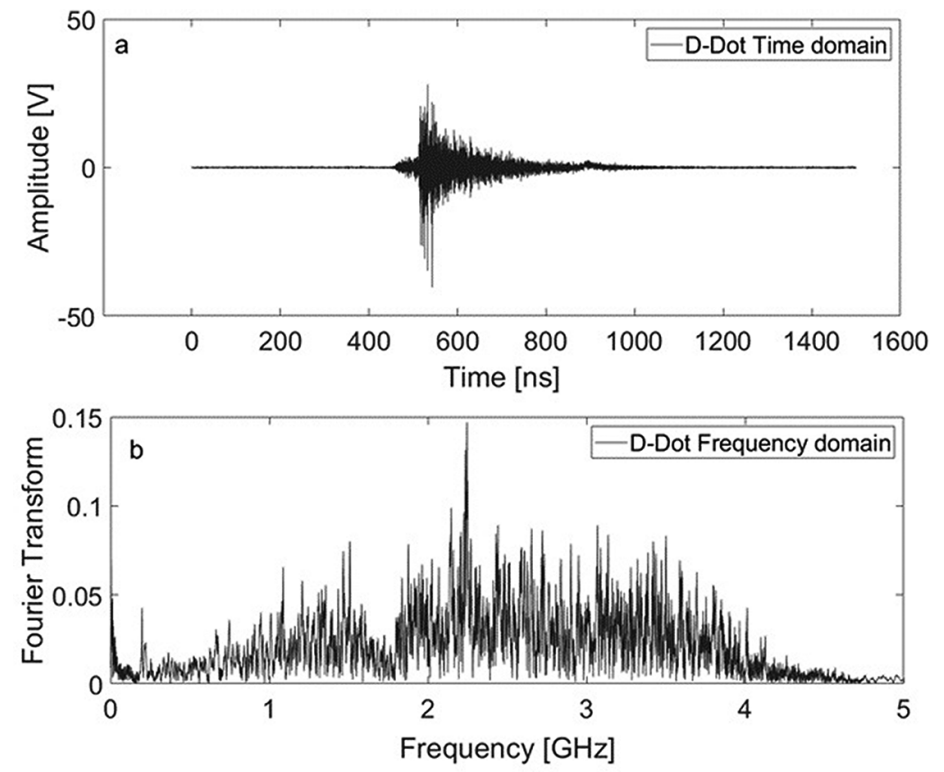

Figure 7. (a) The signal acquired by the D-Dot probe in the time domain. (b) The spectrum retrieved from the reported signal by suitable Fourier transform.

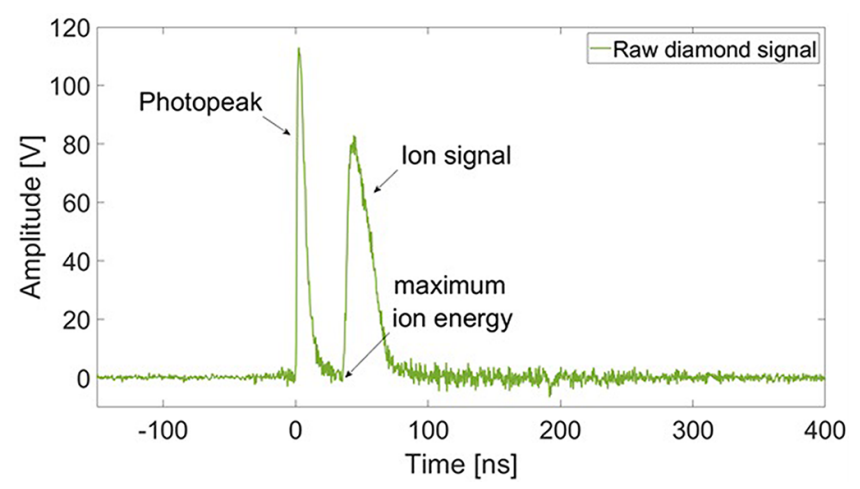

Figure 8. The raw-time domain signal collected by the polycrystalline diamond detector during the described shot.

pipe with a diameter of a few tens of mm mounted on a KF-40 flange on the chamber wall. This would enhance the effective shielding to the produced EMP thanks to the waveguide action performed by the pipe itself ${ }^{[25]}$. Indeed, a pipe having $40 \mathrm{~mm}$ provides a cut-off frequency of $4.39 \mathrm{GHz}$ under which no electromagnetic wave can propagate ${ }^{[8,15,25]}$. Actually, as the pipe is not made of a perfect conductor, there is a steep exponential profile in the frequency domain for the attenuation toward frequencies lower than the cutoff. The same reason causes the electromagnetic waves having frequencies higher than the cutoff to be also attenuated according to the relation $E(z) \propto E_{0} e^{-\alpha_{m n} z}$, where $z$ is the length of the pipe and the coefficient $\alpha_{m n}$ is defined as ${ }^{[25]}$ :

$\alpha_{m n}=\sqrt{\frac{\epsilon}{\mu}} \frac{1}{R_{\mathrm{wg}} \sigma \delta} \sqrt{\frac{f}{f_{\text {cutoff }}}} \frac{1}{\sqrt{1-\frac{f_{\text {cutoff }}^{2}}{f^{2}}}}\left(\xi_{m n}+\eta_{m n}\left(\frac{f_{\text {cutoff }}}{f}\right)^{2}\right)$.
Here, $\epsilon$ and $\mu$ are the electric permittivity and magnetic permeability of the medium filling the waveguide, respectively, $R_{\mathrm{wg}}$ is its radius, $\sigma$ is the conductivity of the pipe, $\delta$ is the associated skin depth, and the dimensionless parameters $\xi_{m n}$ and $\eta_{m n}$ are of the order of unity and depend on the considered propagation mode. In particular, TMmodes have $\xi_{m n}=1$ and $\eta_{m n}=0$, whereas TE modes have $\eta_{m n}=1$ and $\xi_{m n}=1\left(\chi_{m n}^{2^{\prime}}-m^{2}\right)$, where $\chi_{m n}^{\prime}$ is the $n$th root of the derivative of the $m$ th Bessel function.

In Ref. [15] it was shown that without suitable care for EMP issues the diamond signal can be heavily affected by noise due to them, to the point where the photopeak is no more detectable and it is not possible to retrieve any spectra from the stored data. That campaign was performed at the FLAME facility (30 fs pulse, $3 \mathrm{~J}$ maximum energy and $5 \times 10^{19} \mathrm{~W} / \mathrm{cm}^{2}$ intensity on target), where a maximum value of $20 \mathrm{kV} / \mathrm{m}$ was achieved for typical EMPs produced. As reported in Ref. [16], for the FLAME regimes the produced EMPs are expected to be much lower than for the present PHELIX campaign. In fact, as previously mentioned in this manuscript, EMP values up to $100 \mathrm{kV} / \mathrm{m}$ were measured here, about five times higher than for the FLAME campaign. This gives the idea of the high quality of the typical signal shown in Figure 8 that was effectively obtained with the present detector.

The calibrated proton spectrum, shown in Figure 9 together with the associated tolerances, was then retrieved by following the procedure described in Ref. [8]. For this purpose the information on the detector temporal response and CCE obtained during the detector characterization was used, as shown in the previous section. Given the temporal resolution of the system $\Delta t$, the tolerance on the estimated 


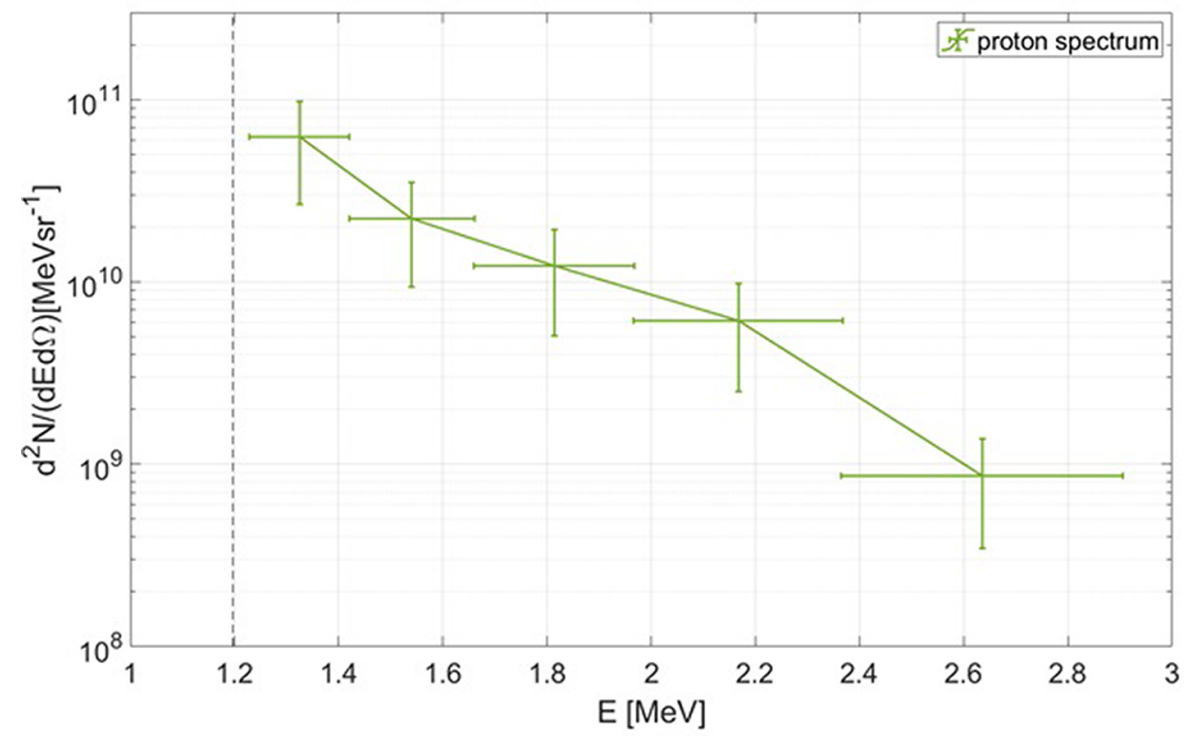

Figure 9. The proton spectrum obtained by the signal in Figure 8. The measured maximum proton energy is $2.6 \pm 0.3 \mathrm{MeV}$. The black dashed line highlights the cutoff energy of the $20 \mu \mathrm{m}$ aluminum filter.

energies is defined as $\frac{\Delta E_{i}}{E_{i}}=2 \sqrt{2} \frac{\Delta t}{d_{\mathrm{TOF}}} \sqrt{\frac{E_{i}}{m_{i}}}[8]$, whereas the one on the number of particles takes into account the CCE variability, the error in estimating the filter attenuation factor, the error in the voltage value provided by the scope and the error related to the integration bin.

The maximum measured proton energy was $2.6 \pm$ $0.3 \mathrm{MeV}$ and, as usual, the number of detected protons decreases for increasing energy. The reason why the observed ion energies are relatively low if compared to the laser energy and intensity on the target has to be found in the main acceleration mechanism. This is not a classical target normal sheath acceleration (TNSA) but rather more a Coulomb explosion of the relativistic plasma channel, formed by the laser interacting with the long scale-length near-critical plasma ${ }^{[32]}$. Moreover, the main purpose of the experimental campaign was to study the enhancement in the electron acceleration exploiting the mechanism of direct laser acceleration ${ }^{[28]}$. The foam thickness was thus not optimized for ion acceleration, and this can explain the observed low energies. The same polycrystalline diamond detector has been recently used in a campaign performed at the VEGA 3 laser on TNSA ion acceleration and was capable of effectively detecting protons with energies up to a few tens of $\mathrm{MeV}^{[33]}$. A Thomson spectrometer was also mounted on a similar angle, and the maximum proton energy detected by the two diagnostics (TOF and Thomson) had a remarkable agreement ${ }^{[33]}$. So, the low maximum energy of the detected protons was physical, and not due to a limitation of the detector that can indeed sense much higher proton energies.

As already mentioned, the information for energies lower than $1.2 \mathrm{MeV}$ was lost due to the presence of the $20 \mu \mathrm{m}$ Al filter used for excluding the contribution of heavier ions,

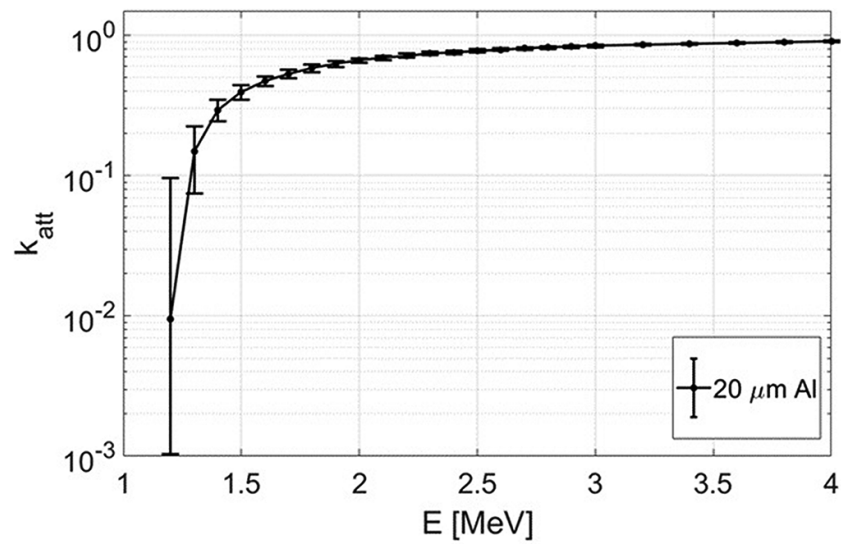

Figure 10. The attenuation coefficient obtained by SRIM computation for the aluminum filter having nominal thickness of $20 \mu \mathrm{m}$.

mounted in place of the inner grid. Nevertheless, the feature of the spectrum for higher energies was retrieved with high accuracy. These particles lost part of their energy on the Al foil, and to reconstruct their accurate spectrum it was necessary to make an estimation of the energy loss for each energy of the incoming particle. This was performed by means of SRIM ${ }^{[34]}$ calculations, and the results for this attenuation are shown in Figure 10. The attenuation factor $k_{\text {att }}\left(E_{\text {in }}\right)$ was obtained by computing the $E_{\text {out }}$ over $E_{\text {in }}$ ratio, where $E_{\text {out }}$ is the value of the energy of a particle with initial energy $E_{\text {in }}$ after crossing the aluminum filter having nominal thickness of $20 \mu \mathrm{m}$. Each $k_{\text {att }}$ value was obtained by Monte Carlo SRIM simulation by averaging the number of total events and the reported uncertainty is given by the standard error. As expected, the uncertainty grows when approaching the filter cutoff, which for protons is $1.2 \mathrm{MeV}$. The range of these particles is in fact almost equal to the filter thickness. 
They are almost stopped inside the aluminum foil and, due to the associated straggling, the spread in the $E_{\text {out }}$ values is higher with respect to more energetic particles, whose range is sensibly larger than the filter thickness.

\section{Conclusions}

In the last decades the field of laser-matter interaction underwent a rapid expansion. The growing availability of powerful laser systems paved the way for the application of laser-driven ion sources to different scenarios, from inertial confinement fusion to material analysis for security and cultural heritage ${ }^{[35-38]}$. This growth needs to be accompanied by the development of suitable diagnostic systems able to effectively characterize the interaction. To do this, it is crucial to face some challenges, for instance to work at a high repetition rate while maintaining a high sensitivity of the measurements, often hindered by the remarkable EMP fields typical of these scenarios.

In this work, we have described a novel detector design based on a $150 \mu \mathrm{m}$ thick polycrystalline diamond sensor having a surface of $15 \mathrm{~mm} \times 15 \mathrm{~mm}$, characterized by a fast temporal response (FWHM $=4.1 \mathrm{~ns}$ ) and a CCE of approximately $42 \%$. This was employed in a TOF scheme during an experimental campaign held at the PHELIX laser facility at high pulse intensity and energy. This is recognized to be one of the main configurations where produced EMP fields are most intense ${ }^{[16]}$ and in these conditions the detector assembly effectively proved its high EMP rejection. As a result, it was thus capable of providing high sensitivity together with a fast temporal response. According to Ref. [16], EMP levels scale with laser energy and mostly with laser intensities. The parameters used in the present campaign gave a significant number of generated EMPs, up to $100 \mathrm{kV} / \mathrm{m}$ at approximately $1 \mathrm{~m}$ distance from the target chamber center (TCC ${ }^{[31]}$. Of course, EMPs up to the MV/m order can be expected at the same distance from the TCC for future experiments with laser intensities increased up to $10^{21} \mathrm{~W} / \mathrm{cm}^{2}$ and beyond. Nevertheless, the present detector has demonstrated a very high rejection to the EMP fields and has the potential to supply high signal-to-noise ratios of the acquired signals and thus accurate real-time spectral characterization of ions in these extreme regimes.

The characteristics of this detector, in particular its high sensitivity, make it perfectly suitable to monitor processes characterized by low fluxes such as, for instance, the $\alpha$ particles produced by laser-initiated $\mathrm{p}^{11} \mathrm{~B}$ fusion reactions ${ }^{[14]}$ or laser interactions with gas-jet targets. Moreover, thanks to the compactness and the easy handling of the detector structure, it is also possible to position a set of them at different angles and distances from the interaction point. This would allow one to obtain simultaneous measurements of the accelerated ions along different directions and to have a more complete reconstruction of the interaction process.

\section{Acknowledgment}

The work has been carried out within the framework of the EUROfusion Consortium and has received funding from the Euratom research and training program 2014-2018 and 2019-2020 under grant agreement No. 633053. The views and opinions expressed herein do not necessarily reflect those of the European Commission.

The results presented here are based on experiment P176, which was performed at the PHELIX facility at the GSI Helmholtzzentrum fuer Schwerionenforschung, Darmstadt (Germany) in the framework of FAIR Phase-0.

The research leading to these results has received funding from LASERLAB-EUROPE (grant agreement No. 654148, European Union's Horizon 2020 research and innovation program).

The research of N.E.A. was supported by the Ministry of Science and Higher Education of the Russian Federation (Agreement with Joint Institute for High Temperatures RAS No. 075-15-2020-785, dated 23 September 2020).

\section{References}

1. D. Margarone, L. Torrisi, S. Cavallaro, E. Milani, G. VeronaRinati, M. Marinelli, C. Tuvè, L. Láska, J. Krása, M. Pfeifer, E. Krouský, J. Ullshmied, L. Ryć, A. Mangione, and A. M. Mezzasalma, Radiat. Eff. Defects Solids 163, 463 (2008)

2. D. Margarone, J. Krása, L. Giuffrida, A. Picciotto, L. Torrisi, T. Nowak, P. Musumeci, A. Velyhan, J. Prokůpek, L. Láska, T. Mocek, J. Ullschmied, and B. Rus, J. Appl. Phys. 109, 103302 (2011).

3. G. Bertuccio, D. Puglisi, L. Torrisi, and C. Lanzieri, Appl. Surf. Sci. 272, 128 (2013).

4. V. Scuderi, G. Milluzzo, D. Doria, A. Alejo, A. G. Amico, N. Booth, G. Cuttone, J. S. Green, S. Kar, G. Korn, G. Larosa, R. Leanza, P. Martin, P. McKenna, H. Padda, G. Petringa, J. Pipek, L. Romagnani, F. Romano, A. Russo, F. Schillaci, G. A. P. Cirrone, D. Margarone, and M. Borghesi, Nucl. Instrum. Methods Phys. Res. Sect. A 978, 164364 (2020).

5. M. Marinelli, E. Milani, G. Prestopino, C. Verona, G. VeronaRinati, M. Cutroneo, L. Torrisi, D. Margarone, A. Velyhan, J. Krasa, and E. Krousky, Appl. Surf. Sci. 272, 104 (2013).

6. R. D. Angelis, F. Consoli, C. Verona, G. D. Giorgio, P. Andreoli, G. Cristofari, M. Cipriani, F. Ingenito, M. Marinelli, and G. Verona-Rinati, J. Instrum. 11, C12048 (2016).

7. M. Cipriani, F. Consoli, P. L. Andreoli, D. Batani, A. Bonasera, G. Boutoux, F. Burgy, G. Cristofari, R. D. Angelis, G. D. Giorgio, J. E. Ducret, A. Flamigni, D. Giulietti, K. Jakubowska, C. Verona, and G. Verona-Rinati, J. Instrum. 14, C01027 (2019).

8. M. Salvadori, F. Consoli, C. Verona, M. Cipriani, M. P. Anania, P. L. Andreoli, P. Antici, F. Bisesto, G. Costa, G. Cristofari, R. De Angelis, G. Di Giorgio, M. Ferrario, M. Galletti, D. Giulietti, M. Migliorati, R. Pompili, and A. Zigler, Sci. Rep. 11, 3071 (2021).

9. H. Daido, M. Nishiuchi, and A. S. Pirozhkov, Rep. Prog. Phys. 75, 056401 (2012).

10. G. Milluzzo, V. Scuderi, A. Alejo, A. G. Amico, N. Booth, M. Borghesi, G. A. P. Cirrone, G. Cuttone, D. Doria, J. Green, S. Kar, G. Korn, G. Larosa, R. Leanza, D. Margarone, P. Martin, P. McKenna, G. Petringa, J. Pipek, L. Romagnani, F. 
Romano, A. Russo, and F. Schillaci, Rev. Sci. Instrum. 90, 083303 (2019).

11. S. Vallières, M. Salvadori, P. Puyuelo-Valdes, S. Payeur, S. Fourmaux, F. Consoli, C. Verona, E. d'Humières, M. Chicoine, S. Roorda, F. Schiettekatte, and P. Antici, Rev. Sci. Instrum. 91, 103303 (2020).

12. R. S. Sussmann, CVD Diamond for Electronic Devices and Sensors (Wiley, Chichester, 2009).

13. C. Verona, M. Marinelli, S. Palomba, G. Verona-Rinati, M. Salvadori, F. Consoli, M. Cipriani, P. Antici, M. Migliorati, F. Bisesto, and R. Pompili, J. Instrum. 15, C09066 (2020).

14. F. Consoli, R. De Angelis, P. Andreoli, A. Bonasera, M. Cipriani, G. Cristofari, G. Di Giorgio, D. Giulietti, and M. Salvadori, Front. Phys. 8, 561492 (2020).

15. M. Salvadori, F. Consoli, C. Verona, M. Cipriani, P. L. Andreoli, G. Cristofari, R. D. Angelis, G. D. Giorgio, D. Giulietti, M. P. Anania, F. Bisesto, G. Costa, M. Ferrario, M. Galletti, R. Pompili, A. Zigler, P. Antici, and M. Migliorati, J. Instrum. 15, C10002 (2020).

16. F. Consoli, V. T. Tikhonchuk, M. Bardon, P. Bradford, D. C. Carroll, J. Cikhardt, M. Cipriani, R. J. Clarke, T. E. Cowan, C. N. Danson, R. De Angelis, M. De Marco, J.-L. Dubois, B. Etchessahar, A. L. Garcia, D. I. Hillier, A. Honsa, W. Jiang, V. Kmetik, J. Krása, Y. Li, F. Lubrano, P. McKenna, J. MetzkesNg, A. Poyé, I. Prencipe, P. Raçczka, R. A. Smith, R. Vrana, N. C. Woolsey, E. Zemaityte, Y. Zhang, Z. Zhang, B. Zielbauer, and D. Neely, High Power Laser Sci. Eng. 8, e22 (2020).

17. F. Consoli, P. L. Andreoli, M. Cipriani, G. Cristofari, R. De Angelis, G. Di Giorgio, L. Duvillaret, J. Krása, D. Neely, M. Salvadori, M. Scisciò, R. A. Smith, and V. T. Tikhonchuk, Philos. Trans. Royal Soc. A Math. Phys. Eng. Sci. 379, 20200022 (2021).

18. E. Woryna, P. Parys, J. Wołowski, and W. Mróz, Laser Particle Beams 14, 293 (1996).

19. A. Higginson, R. J. Gray, M. King, R. J. Dance, S. D. R. Williamson, N. M. H. Butler, R. Wilson, R. Capdessus, C. Armstrong, J. S. Green, S. J. Hawkes, P. Martin, W. Q. Wei, S. R. Mirfayzi, X. H. Yuan, S. Kar, M. Borghesi, R. J. Clarke, D. Neely, and P. McKenna, Nat. Commun. 9, 724 (2018).

20. J. Forneris, A. Lo Giudice, P. Olivero, F. Picollo, A. Re, M. Marinelli, F. Pompili, C. Verona, G. Verona Rinati, M. Benetti, D. Cannata, and F. Di Pietrantonio, Europhys. Lett. 108, 18001 (2014).

21. P. J. Sellin, A. Lohstroh, A. W. Davies, A. Galbiati, J. Parkin, S. G. Wang, and A. Simon, Nucl. Instrum. Methods Phys. Res. Sect. B 260, 293 (2007).

22. S. G. Wang, Q. Zhang, S. F. Yoon, and J. Ahn, Mater. Res. Bullet. 37, 1033 (2002).

23. M. Marinelli, E. Milani, A. Paoletti, A. Tucciarone, G. Verona Rinati, M. Angelone, and M. Pillon, J. Appl. Phys. 89, 1430 (2001).
24. R. Gunnarsson and M. Backstrom, in 2015 IEEE International Symposium on Electromagnetic Compatibility (2015), p. 479.

25. N. Marcuvitz, Waveguide Handbook (McGraw-Hill Book, New York, 1951).

26. V. Bagnoud, B. Aurand, A. Blazevic, S. Borneis, C. Bruske, B. Ecker, U. Eisenbarth, J. Fils, A. Frank, E. Gaul, S. Goette, C. Haefner, T. Hahn, K. Harres, H.-M. Heuck, D. Hochhaus, D. H. H. Hoffmann, D. Javorková, H.-J. Kluge, T. Kuehl, S. Kunzer, M. Kreutz, T. Merz-Mantwill, P. Neumayer, E. Onkels, D. Reemts, O. Rosmej, M. Roth, T. Stoehlker, A. Tauschwitz, B. Zielbauer, D. Zimmer, and K. Witte, Appl. Phys. B 100, 137 (2010).

27. V. Bagnoud and F. Wagner, High Power Laser Sci. Eng. 4, e39 (2016).

28. O. N. Rosmej, M. Gyrdymov, M. M. Günther, N. E. Andreev, P. Tavana, P. Neumayer, S. Zähter, N. Zahn, V. S. Popov, N. G. Borisenko, A. Kantsyrev, A. Skobliakov, V. Panyushkin, A. Bogdanov, F. Consoli, X. F. Shen, and A. Pukhov, Plasma Phys. Control Fusion. 62, 115024 (2020).

29. N. G. Borisenko, A. M. Khalenkov, V. Kmetik, J. Limpouch, Y. A. Merkuliev, and V. G. Pimenov, Fusion Sci. Technol. 51, 655 (2007).

30. F. Consoli, R. De Angelis, T. S. Robinson, S. Giltrap, G. S. Hicks, E. J. Ditter, O. C. Ettlinger, Z. Najmudin, M. Notley, and R. A. Smith, Sci. Rep. 9, 8551 (2019).

31. M. Scisciò, F. Consoli, M. Salvadori, N. Andreev, N. Borisenko, S. Zähter, and O. Rosmej, High Power Laser Sci. Eng. 9, e64 (2021).

32. A. Pukhov, Z.-M. Sheng, and J. Meyer-ter-Vehn, Phys. Plasmas 6, 2847 (1999).

33. M. Salvadori, P. L. Andreoli, M. Cipriani, G. Cristofari, R. De Angelis, S. Malko, L. Volpe, J. A. Perez Hernandez, J. I. Alpinaniz, A. Morace, P. Antici, M. Migliorati, G. Di Giorgio, and F. Consoli, arXiv:2112.10674 (2021).

34. J. F. Ziegler, M. D. Ziegler, and J. P. Biersack, Nucl. Instrum. Methods Phys. Res. Sect. B 268, 1818 (2010).

35. M. Barberio, S. Veltri, M. Scisciò, and P. Antici, Sci. Rep. 7, 40415 (2017).

36. M. Passoni, F. M. Arioli, L. Cialfi, D. Dellasega, L. Fedeli, A. Formenti, A. C. Giovannelli, A. Maffini, F. Mirani, A. Pazzaglia, A. Tentori, D. Vavassori, M. Zavelani-Rossi, and V. Russo, Plasma Phys. Control. Fusion 62, 014022 (2020).

37. P. Puyuelo-Valdes, S. Vallières, M. Salvadori, S. Fourmaux, S. Payeur, J.-C. Kieffer, F. Hannachi, and P. Antici, Sci. Rep. 11, 9998 (2021).

38. L. Giuffrida, F. Belloni, D. Margarone, G. Petringa, G. Milluzzo, V. Scuderi, A. Velyhan, M. Rosinski, A. Picciotto, M. Kucharik, J. Dostal, R. Dudzak, J. Krasa, V. Istokskaia, R. Catalano, S. Tudisco, C. Verona, K. Jungwirth, P. Bellutti, G. Korn, and G. A. P. Cirrone, Phys. Rev. E 101, 013204 (2020). 